\title{
Achieving Full Diversity by Selection in Arbitrary Multi-hop Amplify-and-Forward Relay Networks
}

\author{
Wei Shi and Sumit Roy \\ Dept. of Electrical Engineering, University of Washington \\ Seattle, WA 98195 \\ Email: \{shiwei,sroy\}@u.washington.edu
}

\begin{abstract}
Although several cooperative diversity strategies have been proposed for simple 2-hop multiple-relay networks, practical and efficient strategies that guarantee maximum diversity order for arbitrary, multi-hop amplify-and-forward relay networks are not yet available. In this paper, the maximum achievable diversity order for a given connectivity is shown to be determined by the number of links on the minimum cut set, and achieved by a selection strategy which maximizes end-to-end SNR. The optimal selection requires the channel information of all the links and serves as a performance bound. A sub-optimal selection method is then proposed to achieve full diversity in a distributed and low-complexity manner. The performance loss of the sub-optimal selection is shown to be small by both theoretic analysis and simulations.
\end{abstract}

\section{INTRODUCTION}

In wireless networks, cooperative diversity may be achieved via node collaboration with relay nodes [1]-[3]. A key issue is understanding the maximum achievable diversity in arbitrary ad-hoc networks and subsequently, design practical (distributed and computationally efficient) schemes to achieve this full diversity. While most previous works design focus on relaying methods for two-hop relay networks, in this paper, we address the problem of node selection to achieve maximum diversity order for arbitrary multi-hop relay amplify-and-forward (AF) networks.

For two-hop multiple relay networks, many cooperative diversity protocols have been proposed. Specifically, the orthogonal transmission scheme [3], beamforming techniques [2], distributed space-time coding (DSTC) [4] [5] and node selection [6] [7] are noteworthy. Orthogonal transmission suffers from a bandwidth penalty; beamforming incurs complexity and cost based on needed modifications to existing RF front-ends; DSTC requires synchronization at the packet level, and the code design is difficult and still largely an open research field. Selection diversity, based on instantaneous channel measurements is thus an attractive alternative for its potential to balance simplicity and performance.

When extending from 2-hop to general multi-hop networks with arbitrary number of relays, the aforementioned diversity-achieving schemes (orthogonal transmission, beamforming and DSTC) encounter difficulties for two main reasons. First, these techniques can only be employed by assuming that the maximum diversity order is already known. For example, the orthogonal transmission scheme achieves diversity order of $d$ by orthogonally transmitting over $d$ independent paths, where $d$ is the maximum achievable diversity order. For the DSTC, the code design process varies with the degrees of spatial freedoms. Second, these schemes do not scale to large networks. Consider again the orthogonal transmission scheme as an example. Since choice of $d$ independent paths depend on the network, a central node is needed for decision making. As the number of relays or the number of links becomes larger, higher algorithmic complexity will be incurred at this decision node.

In this paper, we investigate the problem of selecting the best multi-hop AF path with the maximal end-to-end SNR (which we refer to as the optimal selection), and propose a sub-optimal node selection scheme. Both of the optimal and sub-optimal selection schemes are shown to achieve the maximum achievable diversity order a-priori knowledge. The sub-optimal selection scheme achieves the same performance as the optimal selection at high SNR. Both the proposed schemes are distributed, applicable to arbitrary networks and are robust to configuration change. Further, each node only needs to know its adjacent instantaneous channel quality and adjacent connectivity.

This paper is organized as follows. Section II describes the system model for AF relay networks. In Section III, we study the maximum achievable diversity order, and analyzes the outage probability under the optimal selection. Section IV proposes the sub-optimal selection scheme, and studies the performance loss between the optimal and sub-optimal selections. Simulation result is given in Section V. Finally, Section VI concludes the paper.

\section{Selection for Arbitrary AF Relay Networks}

We consider a network, comprising a source (node 1), several relays (node $k, k=2, \cdots, N-1$ ) and a destination node (node $N$ ), connected by an arbitrary network. Each node has a single antenna, and maximal transmit power for the $i$-th node is constrained by $P_{i}$. The channel gain between the $i$-th and $j$-th node are denoted as $\gamma_{i j}=\left|h_{i j}\right|^{2}$, where $h_{i j}$ represents the impulse response of the channel. The channels are assumed independent of each other and modeled as quasistatic fading, implying that the channel realization remains unchanged during the transmission of one message; a new independent realization is then applied to the next message. We assume the channels obey Rayleigh fading distribution, i.e, $h_{i j} \sim \mathcal{C N}\left(0, \frac{1}{\lambda_{i j}}\right)$, where $\lambda_{i j}$ is hazardous rate of distribution of $\gamma_{i j}$. 


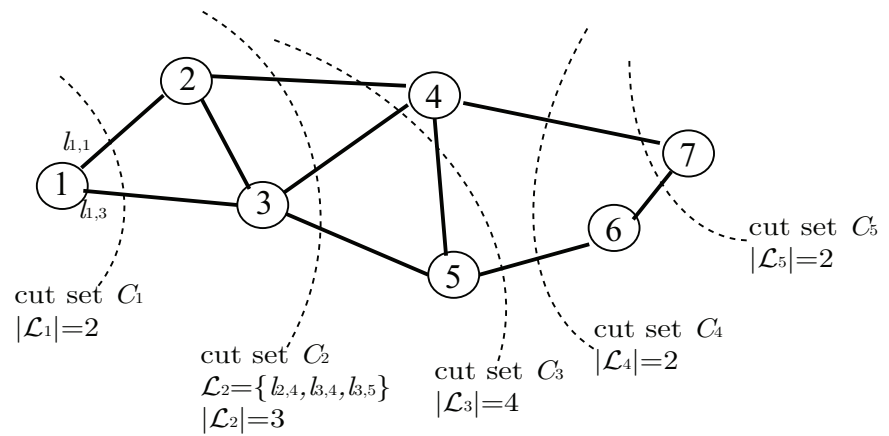

Fig. 1. An example of multi-hop AF relay system with arbitrary connectivity.

We seek to select an end-to-end "best" path with maximal SNR prior to the transmission, based only on network topology information; subsequent data transmission takes place on this selected multi-hop path. We assume half-duplex channels and amplify-and-forward protocol at relay nodes. Each node on the selected path sequentially forwards the source signal using AF protocol. The $(k+1)$-th node on the selected path receives an amplified signal copy of the $k$-th node plus noise, that is,

$$
r_{k+1}=h_{k} \beta_{k} r_{k}+n_{k}
$$

where $h_{k}$ represents the channel, and $n_{k}$ is an additive noise at the $(k+1)$-th node, of mean zero and variance $N_{k} . \beta_{k}$ is a gain control factor at each node in the $k$-th hop to ensure the transmit power is $P_{k}$ via

$$
\beta_{k}=\sqrt{\frac{P_{k}}{E\left\{\left|r_{k}\right|^{2}\right\}}} .
$$

The end-to-end SNR of 2-hop transmission, denoted by $\mathrm{SNR}_{2 \mathrm{hop}}$, is

$$
\mathrm{SNR}_{2 \text { hop }}=\frac{\frac{P_{1} \gamma_{1}}{N_{1}} \frac{P_{2} \gamma_{2}}{N_{2}}}{\frac{P_{1} \gamma_{1}}{N_{1}}+\frac{P_{2} \gamma_{2}}{N_{2}}+1}=\frac{S_{1} S_{2}}{S_{1}+S_{2}+1},
$$

where $\gamma_{k}=\left|h_{k}\right|^{2}$ represents channel gain between the $k$ th and $(k+1)$-th node, where $S_{i}$ denotes the SNR at the receiver in the $i$-th hop. The end-to-end SNR of 3-hop transmission is thus given by

$$
\mathrm{SNR}_{3 \text { hop }}=\frac{S_{1} S_{2} S_{3}}{S_{1} S_{2}+S_{1} S_{3}+S_{2} S_{3}+S_{1}+S_{2}+S_{3}+1}
$$

Similar to the expression of (3) and (4), the end-to-end SNR of $n$-hop transmission is expressed in the form of (8).

In order to study the asymptotic performance as the common transmit power of all nodes is scaled, we let $P_{k}=P$, thereby allowing us to express $S_{1} \cdots S_{n}$ in terms of the normalized variables $\tilde{S}_{1} \cdots \tilde{S}_{n}$ as follows:

$$
\begin{aligned}
& S_{1}=P \tilde{S}_{1}=P\left(\frac{\gamma_{1}}{N_{1}}\right) \\
& S_{2}=P \tilde{S}_{2}=P\left(\frac{P_{2}}{P_{1}} \frac{\gamma_{2}}{N_{2}}\right), \cdots .
\end{aligned}
$$

This allows us to rewrite (8) in the form (9) which is a polynomial in $P$.

Let $\Omega=\left\{\omega_{1}, \omega_{2}, \cdots\right\}$ be the set of all possible end-toend paths, and $\omega_{i}$ be a specific end-to-end path. Now that we have the formulation of the $n$-hop end-to-end SNR, we can express the outage of the optimal selection as

$$
P_{\text {out }}=\operatorname{Pr}\left[\max _{\omega_{i} \in \Omega} \operatorname{SNR}\left(\omega_{i}\right)<\alpha\right],
$$

where $\operatorname{SNR}\left(\omega_{i}\right)$ represent the end-to-end $S N R$ on the $i$-th path, and $\alpha$ is a SNR threshold.

\section{Node Selection: Achieving Max Diversity}

In this section, we show that the diversity order for a given network is bounded by the minimum cut set (i.e. cut set with lowest cardinality). Given a network topology, it well known that the min-cut can be obtained using FordFulkerson algorithm. Hence the optimal route (best end-toend SNR) will achieve the diversity order corresponding to the cardinality of the min-cut.

\section{A. Diversity Order and Cut-set bound}

Given a network graph, denote the complete set of distinct cuts by $\mathcal{C}=\left\{C_{1}, C_{2}, \cdots\right\}$, where $C_{i}$ is a cut separating the source and the destination. Let $\mathcal{L}_{i}$ denote the set of links crossing $C_{i}$, and let $l_{j, k}$ denote the link between the nodes $j$ and $k$. The size of the $i$-th cut $C_{i}$ is $\left|\mathcal{L}_{i}\right|$, the cardinality of $\mathcal{L}_{i}$. For example, in Fig. $1, C_{2}$ is the cut that divides the network into two sub-sets, i.e. $\left\{V_{1}, V_{2}, V_{3}\right\}$ and $\left\{V_{4}, \cdots, V_{7}\right\}$. There are two links crossing the cut $C_{2}$, namely, $\mathcal{L}_{2}=\left\{l_{2,4}, l_{3,4}, l_{3,5}\right\}$.

The upper bound of the maximum diversity order is presented in the following lemma. The upper bound is valid regardless of what relaying schem is used.

Lemma 1: The diversity order is upper bounded by

$$
d \leq \min \left|\mathcal{L}_{i}\right| \text {. }
$$

Proof: We will use the example in Fig. 1 for illustration. For a given cut set, e.g., $C_{2}$ in Fig. 1. Let the source and the destination signals be $x$ and $r_{7}$, and the transmitted and received signals at the boundary nodes (node $2,3,4,5$ ) of $C_{2}$ be $x_{2}, x_{3}, r_{4}, r_{5}$, irrespective of the specifics of the relay scheme. By the information theoretic cut-set bound [12], the mutual information between the source and the destination is bounded by

$I\left(x ; r_{7} \mid h_{1,1}, h_{1,2}, \cdots, h_{6,7}\right) \leq I\left(x_{2}, x_{3} ; r_{4}, r_{5} \mid h_{2,4}, h_{3,4}, h_{3,5}\right)$.

Therefore the outage probability using this relaying scheme is

$$
\begin{aligned}
P_{\text {out }} & =\operatorname{Pr}\left[I\left(x ; r_{7} \mid h_{1,1}, h_{1,2}, \cdots, h_{6,7}\right)<R\right] \\
& \geq \operatorname{Pr}\left[I\left(x_{2}, x_{3} ; r_{4}, r_{5} \mid h_{2,4}, h_{3,4}, h_{3,5}\right)<R\right]
\end{aligned}
$$




$$
\begin{gathered}
\mathrm{SNR}_{n \text { hop }}=\underbrace{\frac{S_{1} S_{2} \cdots S_{n}}{\underbrace{S_{1} S_{2} \cdots S_{n-1}+\cdots}_{\left(\begin{array}{c}
n \\
n-1
\end{array}\right) \text { terms }}}+\underbrace{S_{1} S_{2} \cdots S_{n-2}+\cdots}_{\left(\begin{array}{c}
n \\
n-2
\end{array}\right) \text { terms }}+\cdots+\underbrace{S_{1}+\cdots+S_{n}+1}_{\left(\begin{array}{c}
n \\
1
\end{array}\right) \text { terms }}+1}_{\mathrm{SNR}_{n \text { hop }}=} . \\
\underbrace{P^{n} \tilde{S}_{1} \tilde{S}_{2} \cdots \tilde{S}_{n}}_{\left(\begin{array}{c}
n \\
\left.P^{n-1}\right) \text { terms }
\end{array} \tilde{S}_{1} \tilde{S}_{2} \cdots \tilde{S}_{n-1}+\cdots\right.}
\end{gathered}
$$

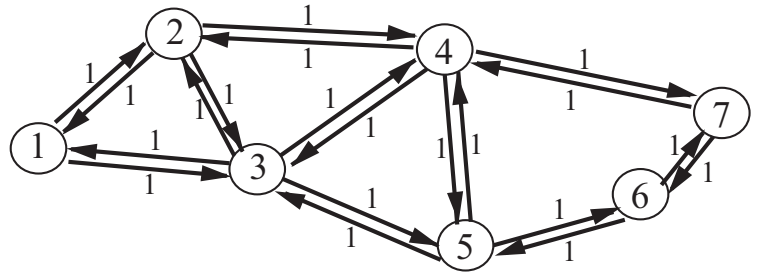

Fig. 2. The bi-directional flow network version of Fig. 1, which is inputted to the Ford-Fulkerson algorithm to find the maximum achievable diversity order.

It is well known that the maximum diversity for a MIMO channel is equal to the number of total channels. For the MIMO channels between \{node 2, node 3\} and \{node 4, node 5$\}$ in Fig. 1, the maximal diversity order is equal to the number of links $\left|\mathcal{L}_{2}\right|$ on the cut-set $C_{2}$.

Applying the above to all cut sets, the maximum achievable diversity is bounded by $\min \left|\mathcal{L}_{i}\right|$.

The well known Ford-Fulkerson algorithm is used to solve the maximum flow problem for a directed flow network, in a polynomial time. The directed flow network has a single source, a destination and multiple intermediate nodes. The edges are directed, and associated with each edge is a capacity.

If we are given a network connectivity, like Fig. 1, we want to find the maximum achievable diversity. First let each link to be bi-directed edges with unity flow capacity as shown in Fig. 2, and then we find a maximum flow by the Ford-Fulkerson algorithm. The value of the maximum flow is equal to the minimal number of links over all cut sets. We can also find the minimum cut set from this maximum flow in a polynomial time.

\section{B. Outage Probability of the Optimal Selection}

In the following, we show that the upper bound of the outage probability of the optimal selection is on the order of $\frac{1}{P^{d}}$. This means that the optimal selection scheme achieve the maximum diversity order. Note that the maximum diversity order is automatically achieved, and there is no need to preidentify the diversity order or to pre-identify the minimum cut sets.

Proposition 1: The outage probability of the optimal selection is upper bounded by

$$
\begin{aligned}
& \operatorname{Pr}\left[\max _{\omega_{i} \in \Omega} \operatorname{SNR}\left(\omega_{i}\right)<\alpha\right] \\
& \leq \quad \prod_{\omega_{i} \in \tilde{\Omega}} \operatorname{Pr}\left[\operatorname{SNR}\left(\omega_{i}\right)<\alpha\right] \\
& P \underset{\approx}{\approx}\left[\prod_{\omega_{i} \in \tilde{\Omega}}\left(\lambda_{\omega_{i}(1)}+\cdots+\lambda_{\omega_{i}\left(n_{i}\right)}\right)\right]\left(\frac{\alpha}{P}\right)^{d}
\end{aligned}
$$

where $\tilde{\Omega}$ is a subset of $\Omega$, consisting of $d$ mutually indepedent paths, and $\lambda_{\omega_{i}(j)}$ is the exponential rates of $\tilde{S}$ on the $j$-th hop of the $i$-th path.

Therefore the selection scheme is of diversity order $d$, which is the maximal achievable diversity order.

Proof: We defer a full proof due to space limitations and only provide an illustrative proof for the relay network in Fig. 1.

The upper bound of $P_{\text {out }}$ is based on the fact that there exists $d$ mutually independent paths, if the minimum number of links over all cut set is equal to $d$. For the case in Fig. 1, the minimum number of links over all cut set is 2 , then 2 mutually independent paths can be found. An example of the set of the mutually independent paths is $\tilde{\Omega}=\left\{\omega_{1}, \omega_{2}\right\}=\{1-2-4-7,1-3-5-6-7\}$. The upper bound is given by

$$
\begin{aligned}
& \operatorname{Pr}\left[\max _{\omega_{i} \in \Omega} \operatorname{SNR}\left(\omega_{i}\right)<\alpha\right] \\
&= \operatorname{Pr}\left[\operatorname{SNR}\left(\omega_{1}\right)<\alpha, \operatorname{SNR}\left(\omega_{2}\right)<\alpha, \cdots\right] \\
& \leq \operatorname{Pr}\left[\operatorname{SNR}\left(\omega_{1}\right)<\alpha, \operatorname{SNR}\left(\omega_{2}\right)<\alpha\right] \\
& \stackrel{(a)}{=} \operatorname{Pr}\left[\operatorname{SNR}\left(\omega_{1}\right)<\alpha\right] \operatorname{Pr}\left[\operatorname{SNR}\left(\omega_{2}\right)<\alpha\right] \\
& P \underset{\approx}{\approx}\left(\lambda_{1,2}+\lambda_{2,4}+\lambda_{4,7}\right)\left(\lambda_{1,3}+\lambda_{3,5}+\lambda_{5,6}+\lambda_{6,7}\right)\left(\frac{\alpha}{P}\right)^{2},
\end{aligned}
$$

where $\lambda_{i, j}$ is the exponential rate of the $\tilde{S}$ of the node $i$ to node $j$ link. Equality (a) follows from that $\operatorname{SNR}\left(\omega_{1}\right)$ and $\operatorname{SNR}\left(\omega_{2}\right)$ are independent since $\omega_{1}$ and $\omega_{2}$ are mutually independent. And the asymptotic property when $P \rightarrow \infty$ is from Lemma 1 in [3]. Note that other choices of $\tilde{\Omega}$ is possible, like $\tilde{\Omega}=\{1-2-3-5-6-7,1-3-4-7\}$.

The upper bound of the outage probability are thus on the order of $\frac{1}{P^{2}}$. Therefore the diversity order achieved by the optimal selection scheme meets the maximal diversity order bound indicated by Lemma 1 . 


\section{Sub-optimal Selection}

It is shown in the previous section that the optimal selection will achieve the maximum diversity order. However, the optimal selection requires a central node to gather information regarding link gains and neighbor topology. Also, an exhaustive search algorithm is needed at the central node to compare all possible paths, which is computationally unaffordable when the network size is large. This leads to the natural search for sub-optimal selection approaches that are distributed and have lower computational complexity while still achieving the maximum diversity order.

We start with simplifying the expression of the end-to-end SNR (8). In the denominator of (8), there are $\left(\begin{array}{c}n \\ n-1\end{array}\right)$ terms of $(n-1)$-th order, $\left(\begin{array}{c}n \\ n-2\end{array}\right)$ terms of $(n-2)$-th order, and so on. The sum of the terms of less than $(n-1)$-th order will play an insignificant role, especially at high SNR. By neglecting those terms, we arrive at an approximated end-to-end SNR, similar to harmonic mean,

$$
\begin{aligned}
\mathrm{SNR}^{\prime} n \text { hop } & =\frac{S_{1} S_{2} \cdots S_{n}}{S_{1} S_{2} \cdots S_{n-1}+\cdots+S_{2} \cdots S_{n-1} S_{n}} \\
& =\frac{1}{\frac{1}{S_{1}}+\cdots+\frac{1}{S_{n}}} .
\end{aligned}
$$

Other statistical properties of the harmonic mean approximation to the end-to-end SNR can be found in [9] [10], we only use the asymptotic results at high SNR [3] [8].

The sub-optimal selection algorithm finds a path that maximizes the SNR/ below, i.e.,

$$
\begin{aligned}
& \max _{\omega_{i} \in \Omega} \operatorname{SNR} \prime\left(\omega_{i}\right) \\
= & \max _{\omega_{i} \in \Omega} \frac{1}{\frac{1}{S_{1}}+\cdots+\frac{1}{S_{n}}} \\
= & \frac{1}{\min _{\omega_{i} \in \Omega}\left(\frac{1}{S_{1}}+\cdots+\frac{1}{S_{n}}\right)} .
\end{aligned}
$$

Treating $\frac{1}{S_{i}}$ as a distance metric, (16) is essentially a shortest path algorithm which selects that with the minimal sum of reciprocals of 1-hop SNRs.

\section{A. Performance loss between the optimal and sub-optimal selection}

This subsection characterizes the performance loss between the optimal and sub-optimal selection.

Proposition 2 (loss in instantaneous SNR): Let $\omega_{*}$ be the path found by the optimal selection, and $\omega_{*}^{\prime}$ be the path found by the sub-optimal selection. Then the difference between the instantaneous SNRs on $\omega_{*}$ and $\omega_{*}^{\prime}$ is at most $\frac{1}{2}$.

Proof: From the result of Appendix A, we have

$$
\operatorname{SNR} \prime\left(\omega_{i}\right)>\operatorname{SNR}\left(\omega_{i}\right)>\operatorname{SNR} \prime\left(\omega_{i}\right)-\frac{1}{2},
$$

for any path $\omega_{i}$. Thus,

$$
\begin{aligned}
\operatorname{SNR}\left(\omega_{*}^{\prime}\right) & >\operatorname{SNR}\left(\omega_{*}^{\prime}\right)-\frac{1}{2} \\
& \stackrel{(a)}{\geq} \operatorname{SNR}\left(\omega_{*}\right)-\frac{1}{2} \\
& >\operatorname{SNR}\left(\omega_{*}\right)-\frac{1}{2},
\end{aligned}
$$

where (a) follows from $\omega_{*}^{\prime}=\arg \max _{\omega_{i}} \operatorname{SNR} /\left(\omega_{i}\right)$.

Also, since $\omega_{*}=\arg \max _{\omega_{i}} \operatorname{SNR}\left(\omega_{i}\right)$, we have

$$
\operatorname{SNR}\left(\omega_{*}^{\prime}\right)<\operatorname{SNR}\left(\omega_{*}\right)
$$

Therefore, the instantaneous SNR on the sub-optimal selected path $\omega_{*}^{\prime}$ satisfies

$$
\operatorname{SNR}\left(\omega_{*}\right)-\frac{1}{2}<\operatorname{SNR}\left(\omega_{*}^{\prime}\right)<\operatorname{SNR}\left(\omega_{*}\right) .
$$

As the system power $P \rightarrow \infty$, the end-to-end SNR will also approaches infinity. It can be envisioned that the performance of the optimal and sub-optimal selections will converge, since the difference of the instantaneous SNRs of the two selections remains at most $\frac{1}{2}$. To quantitatively analyze the converging speed of the SNR outage probabilities, we present the following proposition.

Proposition 3 (loss in SNR outage probability): Obviously, using the sub-optimal selection, the probatility of the SNR outage event is larger than that using the optimal selection. However, as $P \rightarrow \infty$, the difference between the probabilities of the end-to-end SNR outage events of the optimal and sub-optimal selection decays faster than $\frac{1}{P^{d}}$, i.e.,

$$
\lim _{P \rightarrow \infty}\left\{\operatorname{Pr}\left[\operatorname{SNR}\left(\omega_{*}^{\prime}\right)<\alpha\right]-\operatorname{Pr}\left[\operatorname{SNR}\left(\omega_{*}\right)<\alpha\right]\right\} P^{d}=0,
$$

where $\alpha$ is a constant representing the SNR threshold.

Proof: We only sketch the main steps of the proof with details in [11].

First, by the result of Appendix A (32), we have

$$
\operatorname{SNR}\left(\omega_{*}^{\prime}\right) \leq \operatorname{SNR}\left(\omega_{*}\right)<\operatorname{SNR} \prime\left(\omega_{*}^{\prime}\right),
$$

which implies

$$
\begin{aligned}
& \operatorname{Pr}\left[\operatorname{SNR}\left(\omega_{*}^{\prime}\right)<\alpha\right] \\
\geq & \operatorname{Pr}\left[\operatorname{SNR}\left(\omega_{*}\right)<\alpha\right] \\
\geq & \operatorname{Pr}\left[\operatorname{SNR}\left(\omega_{*}^{\prime}\right)<\alpha\right] .
\end{aligned}
$$

This suggests an indirect way of proving (21) by proving

$$
\lim _{P \rightarrow \infty}\left\{\operatorname{Pr}\left[\operatorname{SNR}\left(\omega_{*}^{\prime}\right)<\alpha\right]-\operatorname{Pr}\left[\operatorname{SNR} \prime\left(\omega_{*}^{\prime}\right)<\alpha\right]\right\} P^{d}=0 .
$$

Since $\left\{\operatorname{SNR} \prime\left(\omega_{*}^{\prime}\right)<\alpha\right\} \subseteq\left\{\operatorname{SNR}\left(\omega_{*}^{\prime}\right)<\alpha\right\}$, the left hand side of (24) is expressed as

$$
\begin{aligned}
& \operatorname{Pr}\left[\operatorname{SNR}\left(\omega_{*}^{\prime}\right)<\alpha\right]-\operatorname{Pr}\left[\operatorname{SNR} \prime\left(\omega_{*}^{\prime}\right)<\alpha\right] \\
= & \operatorname{Pr}\left[\left\{\operatorname{SNR}\left(\omega_{*}^{\prime}\right)<\alpha\right\} \backslash\left\{\operatorname{SNR} \prime\left(\omega_{*}^{\prime}\right)<\alpha\right\}\right] \\
= & \operatorname{Pr}\left[\left\{\operatorname{SNR}\left(\omega_{*}^{\prime}\right)<\alpha\right\} \cap\left\{\operatorname{SNR} \prime\left(\omega_{*}^{\prime}\right)>\alpha\right\}\right] \\
= & \operatorname{Pr}\left[\operatorname{SNR}\left(\omega_{*}^{\prime}\right)<\alpha<\operatorname{SNR}\left(\omega_{*}^{\prime}\right)\right],
\end{aligned}
$$


where $A \backslash B$ denotes the set difference between set $A$ and set $B$.

Next, the probability (25) can be expanded as

$$
\begin{aligned}
& \operatorname{Pr} {\left[\operatorname{SNR}\left(\omega_{*}^{\prime}\right)<\alpha<\operatorname{SNR} \prime\left(\omega_{*}^{\prime}\right)\right] } \\
&=\operatorname{Pr}\left[\begin{array}{l}
\left\{\operatorname{SNR}\left(\omega_{1}\right) \geq \operatorname{SNR}\left(\omega_{i}\right), i \neq 1\right\} \\
\cap\left\{\operatorname{SNR}\left(\omega_{1}\right)<\alpha<\operatorname{SNR}\left(\omega_{1}\right)\right\}
\end{array}\right]+ \\
& \operatorname{Pr}\left[\begin{array}{l}
\left\{\operatorname{SNR} \prime\left(\omega_{2}\right) \geq \operatorname{SNR}\left(\omega_{i}\right), i \neq 2\right\} \\
\cap\left\{\operatorname{SNR}\left(\omega_{2}\right)<\alpha<\operatorname{SNR}\left(\omega_{2}\right)\right\}
\end{array}\right]+\cdots
\end{aligned}
$$

We next prove that each term in the above equation decays faster than $\frac{1}{P^{d}}$. Omitting several steps, and without loss of generality, we are able to show the first term in the above equation is bounded by

$$
\begin{aligned}
& \operatorname{Pr}\left[\begin{array}{c}
\left\{\operatorname{SNR} \prime\left(\omega_{1}\right) \geq \operatorname{SNR} \prime\left(\omega_{i}\right), i \neq 1\right\} \\
\cap\left\{\operatorname{SNR}\left(\omega_{1}\right)<\alpha<\operatorname{SNR} \prime\left(\omega_{1}\right)\right\}
\end{array}\right] \\
\leq & \left(\prod_{\omega_{i} \in \tilde{\Omega} \backslash\left\{\omega_{1}\right\}} \operatorname{Pr}\left[\operatorname{SNR}\left(\omega_{i}\right)<\alpha+\frac{1}{2}\right]\right) \\
& \operatorname{Pr}\left[\operatorname{SNR}\left(\omega_{1}\right)<\alpha<\operatorname{SNR} \prime\left(\omega_{1}\right)\right],
\end{aligned}
$$

where $\tilde{\Omega}$ is a set of mutually independent paths. There are $d-1$ terms of $\operatorname{Pr}\left[\operatorname{SNR}\left(\omega_{i}\right)<\alpha+\frac{1}{2}\right]$ in the above equation, and each decays on the order of $\frac{1}{P}$. And $\operatorname{Pr}\left[\operatorname{SNR}\left(\omega_{1}\right)<\alpha<\operatorname{SNR} /\left(\omega_{1}\right)\right]$ decays faster than $\frac{1}{P}$, since

$$
\begin{aligned}
& \lim _{P \rightarrow \infty} P \cdot \operatorname{Pr}\left[\operatorname{SNR}\left(\omega_{1}\right)<\alpha<\operatorname{SNR} \prime\left(\omega_{1}\right)\right] \\
= & \lim _{P \rightarrow \infty} P \cdot \operatorname{Pr}\left[\operatorname{SNR}\left(\omega_{1}\right)<\alpha\right] \\
& -\lim _{P \rightarrow \infty} P \cdot \operatorname{Pr}\left[\operatorname{SNR} \prime\left(\omega_{1}\right)<\alpha\right] \\
= & 0,
\end{aligned}
$$

which follows from asymptotic result of multihop system in [8]. Thus (27) decays faster than $\frac{1}{P^{d}}$.

Therefore the whole propsition is proved that the performance loss in outage probability decays faster than $\frac{1}{P^{d}}$.

We have already shown that the outage probability of the optimal selection decays on the order of $\frac{1}{P^{d}}$. Therefore, by Proposition 3, the sub-optimal selection not only achieves the maximum diversity, but also has no performance loss in terms of SNR outage probability at high SNR.

\section{B. Practical implementation of the sub-optimal selection}

The sub-optimal selection scheme can be implemented in a fully distributed and adaptive manner. It is essentially a distributed shortest path algorithm, requiring tha each nodes only has local information, i.e. its adjacent instantaneous channel gains and 1-hop connectivity. The selection is adaptive in the sense that it adjusts whenever channel conditions or network connectivity changes. The sub-optimal selection algorithm requires the following to be deployed in practice:

1) Channel estimation: For each link, the instantaneous channel gains are estimated by using the received pilot signal strength, and are assumed known by both nodes.

2) Distributed shortest path (Bellman-Ford) algorithm: Each node maintains a routing table, and periodically sends path vector tuples (i.e. destination and the corresponding distance) to its all immediate neighbor nodes. Upon receiving any update, a node updates its routing table in turn until the routing table stabilizes.

3) Data transmission: In the 1st time slot, the source notifies the next node on its routing table (such that the next node stays active) and broadcasts it's data, while other relay nodes remain idle. In the 2 nd time slot, the active relay notifies the third relay node and amplifies-and-forwards the received signal from the 1st slot. The remaining nodes amplify-and-forward in subsequent slots until the data reaches the destination.

4) Periodically or responsively adapt path selection: The channel gains on all the links are measured periodically; the distributed shortest path algorithm will generate new routing tables according to changes in channel condition. Moreover, whenever the connectivity changes (e.g. a node joins or leaves the network), any node that senses the change will trigger path adaptation by sending an updated path vector tuple to their neighbor nodes.

\section{Simulation Results}

In this section, we present simulation results of the optimal and sub-optimal scheme. Fig. 3 shows the network configuration used in the simulation, comprising of a source and a destination, and 7 relays. Each node has power constraint $P$. The channels are assumed independently Rayleigh fading, $h_{i j} \sim \mathcal{C N}\left(0, \frac{1}{\left(d_{i j}\right)^{l_{\alpha} / 2}}\right)$, where $d_{i j}$ is inter-node distance and $l_{\alpha}=4$ is the path loss exponent.

For this network, it is easy to see the minimum number of links over all possible cut sets is 3 . Therefore, by Lemma 1 , the maximum achievable diversity order is 3 .

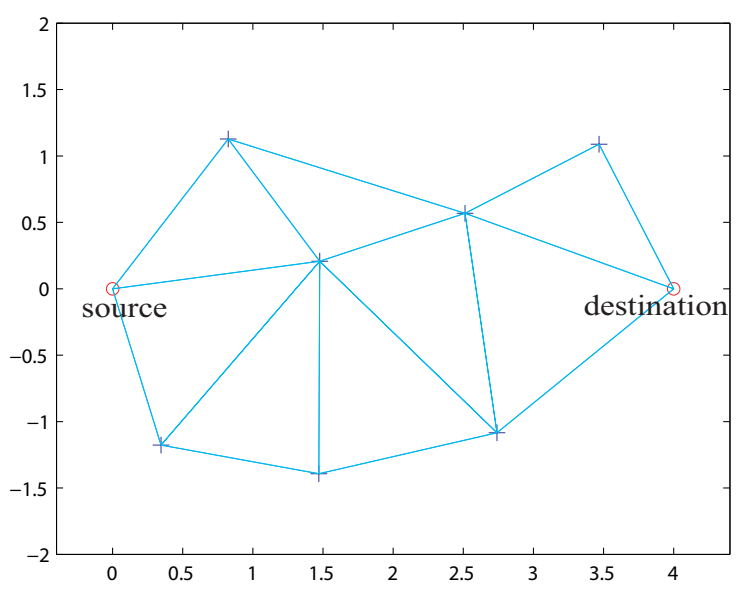

Fig. 3. The network configuration used in the simulation.

We conduct a Monte Carlo simulation to evaluate the bit error rate (BER) of the optimal and sub-optimal selection schemes. In each Monte Carlo run, channel impulse responses are generated independently and obeying Rayleigh 
fading distribution. The optimal and the sub-optimal paths are selected by the optimal instantaneous end-to-end SNR criterion (5) and by the sub-optimal criterion (16). Then on each selected path, an uncoded BPSK symbol is transmitted from the source, being amplified and forwarded by the relays, and finally decoded at the destination. Finally the BER is estimated by the number of decoding failure over the total number of Monte Carlo runs.

Fig. 4 plots the BER as a function of $P$, for the optimal and sub-optimal selection, respectively. Averaging over $10^{6}$ independent runs, we obtain the shown BER versus $P$ curve. At high power region $(P>15 \mathrm{~dB})$, both BERs decay on the order of $\frac{1}{P^{3}}$, conformal to the maximum diversity order as indicated by Lemma 1.

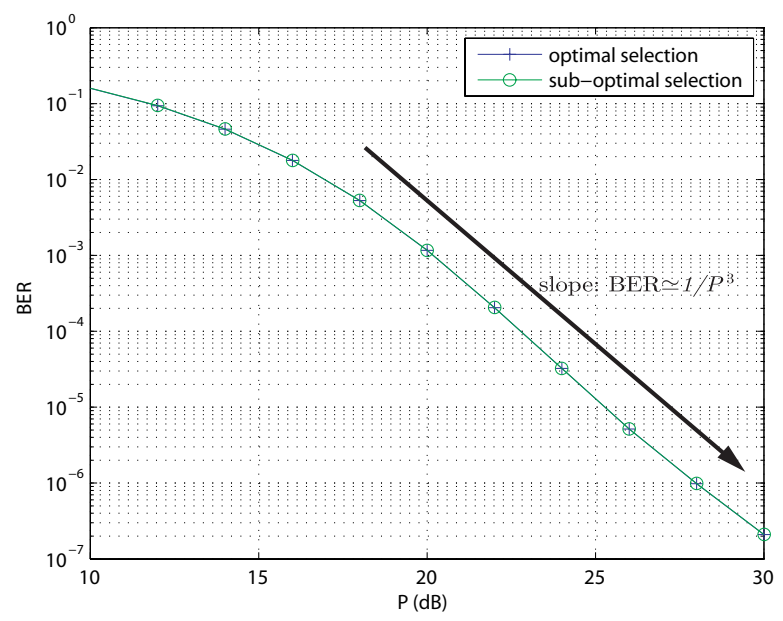

Fig. 4. The BER versus the power constraint $P$ at each node, for the optimal and sub-optimal selection. It is noticed that the slope of BER curve is on the order of $\frac{1}{p^{3}}$, which means diversity order is 3 .

\section{CONCLUSION}

In this paper, we investigated the selection strategy in a multi-hop relay network with arbitrary topology, aimed at maximizing the diversity order. We demonstrated that the maximum achievable diversity order is bounded by the cardinality of the minimum cut set, and that optimal selection can achieve this diversity order. To reduce the amount of information needed and algorithmic complexity, a suboptimal selection is proposed. The performance gap between the optimal and the sub-optimal selection is shown to be small, and is verified by the simulation result.

\section{APPENDIX A}

Let

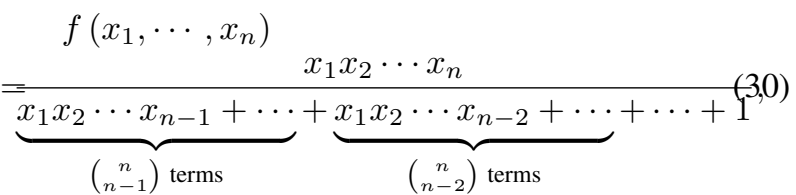

and

$$
=\frac{g\left(x_{1}, \cdots, x_{n}\right)}{\frac{1}{x_{1}}+\frac{1}{x_{2}}+\cdots+\frac{1}{x_{n}}},
$$

and the domains for the function $f$ and $g$ are $x_{i}>0$. We are going to prove

$$
g\left(x_{1}, \cdots, x_{n}\right)-\frac{1}{2}<f\left(x_{1}, \cdots, x_{n}\right)<g\left(x_{1}, \cdots, x_{n}\right) .
$$

It is easily seen that

$$
f\left(x_{1}, \cdots, x_{n}\right)<g\left(x_{1}, \cdots, x_{n}\right) .
$$

Subtracting (30) from (31), the resulting is given by (33). Expanding the denominator of (33) and comparing the expanded denominator with the numerator, it is seen that (33) is less than $\frac{1}{2}$.

Therefore (32) is proved.

\section{REFERENCES}

[1] G. Kramer, M. Gastpar, and P. Gupta, "Cooperative strategies and capacity theorems for relay networks," IEEE Trans. Info. Theory, vol. 51, no. 9, pp. 3037-3063, Sep. 2005.

[2] A. Sendonaris, E. Erkip, and B. Aazhang, "User cooperation diversity. Part I: System description," IEEE Trans. Commun., vol. 51, no. 11, pp. 1927-1928, Nov. 2003.

[3] J. N. Laneman, D. N. C. Tse, and G. W. Wornell, "Cooperative diversity in wireless networks:efficient protocols and outage behavior," IEEE Trans. Info. Theory, vol. 50, no. 12, pp. 3062-3080, Dec. 2004.

[4] J. N. Laneman and G. W. Wornell, "Distributed space-time coded protocols for exploiting cooperative diversity in wireless networks," IEEE Trans. Info. Theory, vol. 49, no. 10, pp. 2415-2525, Oct. 2003.

[5] R. U. Nabar, H. Bolcskei, and F. W. Kneubuhler, "Fading relay channels: Performance limits and space-time signal design," IEEE J. Select. Areas Commun., vol. 22, no. 6, pp. 1099-1109, Jun. 2004.

[6] A. Bletsas, A. Khisti, D. P. Reed, and A. Lippman, "A simple cooperative diversity method based on network path selection," IEEE J. Select. Areas Commun., vol. 24, no. 3, pp. 659-672, Mar. 2006.

[7] Y. Zhao, R. S. Adve, and T. J. Lim, "Improving amplify-and-forward relay networks: Optimal power allocation versus selection," IEEE Trans. Wireless Commun., vol. 6, no. 8, pp. 3114-3123, Aug. 2007.

[8] A. Riberio, X. Cai, and G. B. Giannakis, "Symbol error probabilities for general cooperative links," IEEE Trans. Wireless Commun., vol. 4, no. 3, pp. 1264-1273, May 2005.

[9] M. O. Hasna and M.-S. Alouini, "End-to-end performance of transmission systems with relays over Rayleigh-fading channels," IEEE Trans. Wireless Commun., vol. 2, no. 6, pp. 1126-1131, Nov. 2003.

[10] M. O. Hasna and M.-S. Alouini, "Harmonic mean and end-to-end performance of transmission systems with relays," IEEE Trans. Commun., vol. 52, no. 1, pp. 130-135, Jan. 2004.

[11] W. Shi and S. Roy, "Distributed low-complexity full-diversityachieving selection for arbitrary multi-hop amplify-and-forward relay networks," In Preparation.

[12] T. M. Cover and J. Thomas, Elements of Information Theory. New York:Wiley, 1991. 


$$
g\left(x_{1}, \cdots, x_{n}\right)-f\left(x_{1}, \cdots, x_{n}\right)=\frac{\left(\frac{1}{x_{1} x_{2}}+\cdots+\frac{1}{x_{n-1} x_{n}}\right)+\cdots+\frac{1}{x_{1} x_{2} \cdots x_{n}}}{\left(\frac{1}{x_{1}}+\frac{1}{x_{2}}+\cdots+\frac{1}{x_{n}}\right)\left[\left(\frac{1}{x_{1}}+\cdots+\frac{1}{x_{n}}\right)+\left(\frac{1}{x_{1} x_{2}}+\cdots+\frac{1}{x_{n-1} x_{n}}\right)+\cdots+\frac{1}{x_{1} x_{2} \cdots x_{n}}\right]}
$$

